\title{
Impacto de un curso taller de cinco horas en la interpretación de la espirometría
}

\section{Impact of a 5-hour workshop on the interpretation of spirometry}

\author{
Juan Carlos Vázquez-García, Rebeca Ortiz-Siordia, ${ }^{*}$ Francisco Franco-Marina, ${ }^{*}$ \\ Jorge Salas-Hernández, ${ }^{*}$ Rosaura Esperanza Benítez-Pérez, ${ }^{*}$ Rogelio Pérez-Padilla*
}

*Instituto Nacional de Enfermedades Respiratorias Ismael Cosío Villegas, Ciudad de México, México.

\begin{abstract}
RESUMEN. Introducción: El subdiagnóstico y error diagnóstico del asma y la enfermedad pulmonar obstructiva crónica es muy grande en todo el mundo, en parte, debido al pobre acceso y el uso médico de la espirometría. El objetivo fue evaluar el impacto de un curso-taller presencial en la competencia médica para el uso y la interpretación de la espirometría. Método: Se desarrolló un curso de cinco horas que comprendió conferencias, un video técnico y un taller de interpretación de 16 espirometrías reales. Profesionales médicos de 31 estados completaron el curso y sus evaluaciones inicial y final. Resultados: Un total de 1,343 profesionales, $71.3 \%$ médicos generales y familiares, completaron el curso. Sólo $37 \%$ de los participantes reconocieron de manera correcta a la espirometría con broncodilatador como la prueba de elección diagnóstica y $11.1 \%$ fueron capaces de diagnosticar obstrucción al flujo aéreo grave. Después del curso, se observó una mejoría significativa en los conocimientos y $81.2 \%$ diagnosticaron de forma acertada la obstrucción grave. Conclusión: El nivel de conocimientos y competencia médica para el uso e interpretación de la espirometría fue claramente insuficiente; sin embargo, un curso-taller de cinco horas fue bastante eficiente para fines de capacitación dirigida a la competencia médica.
\end{abstract}

Palabras clave: Espirometría, asma, enfermedad pulmonar obstructiva crónica, primer nivel de atención, educación médica.

\section{INTRODUCCIÓN}

La enfermedad pulmonar obstructiva crónica (EPOC) y el asma en su conjunto causaron 28,551 muertes en México en el año 2016, ${ }^{1}$ la gran mayoría por EPOC, lo que la convierte en la séptima causa de mortalidad general (cuarta en 2015). La prevalencia de EPOC en nuestro país es del $7.8 \%$ de las personas mayores de 40 años mientras que la

Correspondencia:

Dr. Juan Carlos Vázquez García

Instituto Nacional de Enfermedades Respiratorias Ismael Cosío

Villegas, Ciudad de México.

Correo electrónico: drjcvazquez@gmail.com

Trabajo recibido: 11-XII-2018; aceptado: 17-I-2019.
ABSTRACT. Introduction: Underdiagnosis and misdiagnosis of asthma and COPD is enormous worldwide. This is partly explained by a poor accessibility and medical use of spirometry. The aim of this study was to describe the impact of a 5-hour workshop on medical competence regarding use and interpretation of spirometry. Method: Topic sessions, a technical video and 16-real spirometries workshop were developed for the project. Medical professionals from 31 states completed a residential course with initial and final evaluation. Results: A total of 1,343 professionals, $71.3 \%$ general practitioners and family physicians, completed the workshop and examination. Only $37 \%$ were able to recognized post-bronchodilator spirometry as the recommended diagnostic test for COPD and 11\% correctly diagnosed severe airflow obstruction in the baseline evaluation. After the workshop, there was a significant improvement in all items of the examination and $81.2 \%$ correctly recognized severe airflow obstruction. Conclusion: In this study medical knowledge and competence for using and interpreting spirometry in general practice is clearly insufficient, but a few-hours workshop may be highly efficient for training medical competence in continuous medical education programs.

Keywords: Spirometry, asthma, COPD, primary health care, medical education.

de asma es de alrededor de $7.5 \%$ de la población general. ${ }^{2,3}$ Sin embargo, el subdiagnóstico y el error diagnóstico es muy frecuente en ambas enfermedades. Se estima que el subdiagnóstico de EPOC rebasa el 81\% en la población general a nivel mundial, mientras que en México es del $86 \%{ }^{4}{ }^{4}$ La razón de esto se explica en buena parte por un pobre uso de la espirometría, en particular en el primer y segundo nivel de atención..$^{5,6}$ Esta prueba es el estándar de oro para definir obstrucción al flujo aéreo, el componente principal para el diagnóstico de asma y EPOC. ${ }^{7-9} \mathrm{El}$ papel diagnóstico de esta prueba es el mismo que el de la glucemia para la diabetes y el de la medición de presión arterial para la hipertensión arterial. En la actualidad, se han definido las recomendaciones internacionales para la realización e interpretación de la espirometría, ${ }^{10-14}$ que comprende también el consultorio médico, ${ }^{12-14}$ pero su 
implementación y uso es todavía muy bajo en México, al igual que en la mayoría de los países en desarrollo.

La espirometría forzada es una prueba de función pulmonar mecánica; mide el mayor volumen o el flujo de aire que se puede exhalar con máximo esfuerzo, con respecto al tiempo medido en segundos. ${ }^{10,15,16}$ La competencia médica para la interpretación de la espirometría puede simplificarse en un proceso de dos pasos: 1) graduar la calidad técnica con la que se realizó la prueba, que significa definir la confiabilidad para la interpretación y 2) definir el patrón ventilatorio de la espirometría (normal, obstructivo o restrictivo). La mayor utilidad de la espirometría es definir el diagnóstico de obstrucción al flujo aéreo, para lo cual es el estándar dorado. Se diagnóstica cuando la relación entre el principal flujo (volumen espiratorio forzado en un segundo o $\mathrm{FEV}_{1}$ ) y el mayor volumen de aire que se puede exhalar (capacidad vital forzada o FVC), es decir, su cociente FEV $/$ /FVC disminuye por debajo del límite inferior normal. En ausencia de obstrucción bronquial, la disminución de la FVC es compatible con restricción pulmonar (volumen pulmonar disminuido).

La implementación de la espirometría en cualquier nivel de atención es una estrategia fundamental para mejorar la detección y seguimiento de todas las enfermedades pulmonares crónicas. ${ }^{5,6}$ Sin embargo, más allá de la adquisición de espirómetros, se requiere de la capacitación, acreditación y seguimiento del personal que va a realizar la prueba, así como de un programa de educación continua que entrene a todo médico para el uso, la evaluación de la calidad de la prueba y la adecuada interpretación de la misma. ${ }^{10,11}$ El objetivo de este estudio fue describir el proceso de estructuración e implementación, así como el impacto de un curso-taller de cinco horas enfocado en fortalecer la competencia médica para el uso y la interpretación de la espirometría.

\section{MÉTODO}

El curso-taller de interpretación de espirometría (CIE) fue diseñado por iniciativa de los autores como parte del proyecto de educación médica en espirometría de la Asociación Latinoamericana del Tórax (ALAT). Este estudio incluyó el desarrollo de los materiales educativos y la organización de los cursos.

\section{Participantes}

Durante un período de dos años se organizó el CIE en los estados de la República Mexicana y la Ciudad de México. Los participantes en su gran mayoría eran médicos generales y familiares quienes ejercían práctica privada; todos fueron invitados de forma directa por el patrocinador, debido a que se identificaban como médicos que atendían por lo regular pacientes con asma o EPOC.

\section{Diseño y dinámica del curso}

El CIE se estructuró con base en el curso estandarizado para técnicos en espirometría que se imparte en el Instituto Nacional de Enfermedades Respiratorias (INER) Ismael Cosío Villegas desde el año 1995, el cual a su vez es una adaptación y evolución del curso de espirometría del Instituto Nacional de Salud y Seguridad Ocupacional de los Estados Unidos (NIOSH). Se trata de un curso teóricopráctico y de acreditación, con una duración de 16 horas, que se imparte en dos días continuos y que se apega a los estándares internacionales vigentes de la Sociedad Americana del Tórax y de la Sociedad Europea Respiratoria, ATS/ ERS 2005.10,11 El CIE se estructuró por dos especialistas en neumología, expertos en fisiología respiratoria y directores de curso NIOSH (JCVG y RPP) y una pedagoga experta en educación médica (ROS). El objetivo fue fortalecer los conocimientos y la competencia en la interpretación de la espirometría por parte de los médicos. El curso contó con cuatro conferencias con una duración total de dos horas que incluyeron las bases anatómicas y fisiológicas, generalidades de espirometría y una conferencia sobre el proceso de interpretación; además, un video de 15 minutos que muestra en detalle la realización completa de una espirometría, así como un taller de interpretación de dos horas. Se diseñó y editó un manual específico y las presentaciones correspondientes para proyección de cada conferencia y el taller de interpretación. El manual contiene toda la información teórica, todas las ilustraciones y 16 ejercicios para ser completados durante el taller. ${ }^{16}$

Cada curso se impartió por un solo profesor, neumólogo experto; cuando se incorporaba un nuevo profesor, se le requería revisar previamente los materiales y presenciar un curso completo por uno de los profesores autores. Al inicio de cada curso se realizaba un registro de los participantes, quienes eran invitados a completar una encuesta y una evaluación inicial. La encuesta se compuso de 11 preguntas sobre sus datos generales, de práctica profesional y frecuencia con la que usaban herramientas diagnósticas, como espirometría, glucemia y electrocardiograma. Las evaluaciones tanto la inicial como la final contenían tres preguntas de opción múltiple sobre definiciones de los principales parámetros de la espirometría: la FVC y el $\mathrm{FEV}_{1}$. Además, se integró un ejercicio de interpretación estructurada, característica de obstrucción al flujo aéreo de grado grave (figura 1). Los ejercicios del taller consistieron en 16 espirometrías reales de complejidad progresiva, para la identificación de criterios de aceptabilidad ( 5 puntos) y repetibilidad (5 puntos), errores frecuentes, así como ejemplos de los diferentes patrones ventilatorios; se pusieron cuatro ejercicios para identificar el grado de obstrucción al flujo aéreo y la respuesta al broncodilatador. Una vez impartidas las conferencias teóricas y proyectado 


\begin{tabular}{|l|c|c|c|c|c|c|c|}
\hline & & \multicolumn{2}{|c|}{ Maniobra [A] } & \multicolumn{2}{c|}{ Maniobra [B] } & \multicolumn{2}{c|}{ Maniobra [C] } \\
\cline { 5 - 8 } Parámetro & Predichos & Actual & $\%$ Predicho & Actual & \% Predicho & Actual & \% Predicho \\
\hline FVC & $2.22 \mathrm{~L}$ & $1.83 \mathrm{~L}$ & 82.4 & $1.85 \mathrm{~L}$ & 83.3 & $1.76 \mathrm{~L}$ & 79.3 \\
\hline FEV & $1.69 \mathrm{~L}$ & $0.81 \mathrm{~L}$ & 47.9 & $0.76 \mathrm{~L}$ & 45.0 & $0.79 \mathrm{~L}$ & 46.7 \\
\hline $\mathrm{FEV}_{1} / \mathrm{FVC}$ & $77 \%$ & $44.6 \%$ & 57.9 & $41.1 \%$ & 53.4 & $45.1 \%$ & 58.6 \\
\hline PEF & $5.28 \mathrm{~L} / \mathrm{S}$ & $2.06 \mathrm{~L} / \mathrm{S}$ & 39.2 & $2.11 \mathrm{~L} / \mathrm{S}$ & 40.0 & $2.13 \mathrm{~L} / \mathrm{S}$ & 40.3 \\
\hline
\end{tabular}

$\mathrm{FEV}_{1}$ : volumen espiratorio forzado en un segundo, FVC: capacidad vital forzada, PEF: flujo espiratorio pico.
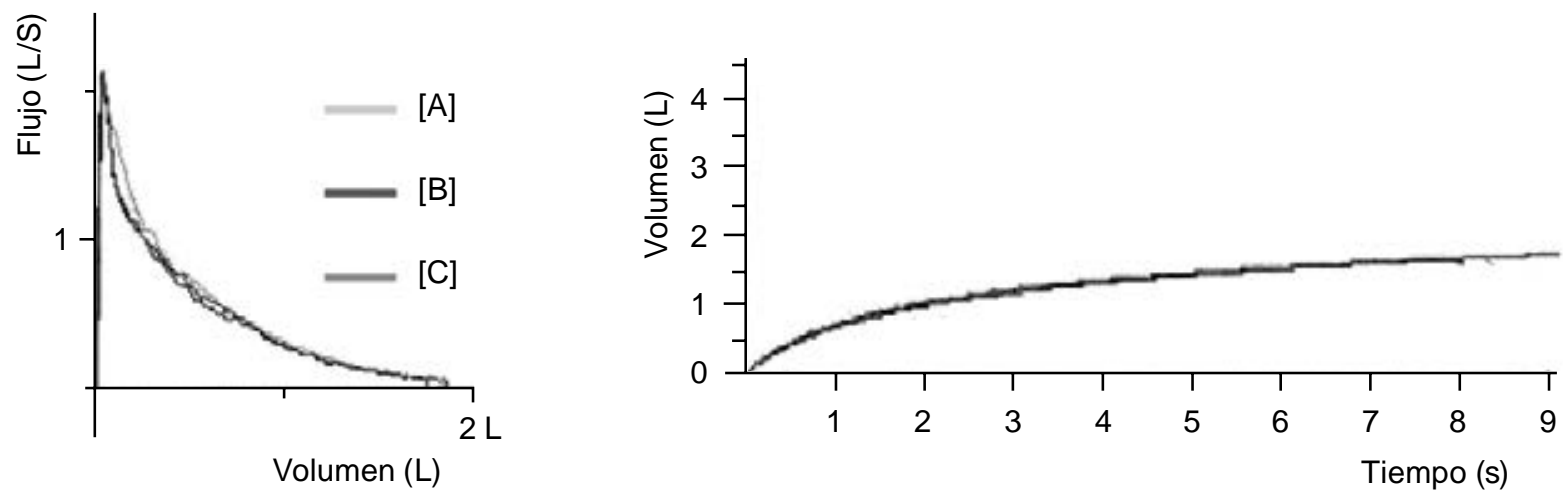

Figura 1: Se muestran los parámetros de las tres maniobras de espirometría y las gráficas de flujo-volumen y volumen tiempo. El diagnóstico de obstrucción se establece con base en una relación $\mathrm{FEV}_{1} / \mathrm{FVC}$ de sólo $44.6 \%$ y de grado grave por un valor de $\mathrm{FEV}_{1}$ menor al $50 \%$ (47.9\%).

el video, la dinámica del taller consistía en proyectar una espirometría y los asistentes completaban de forma individual la interpretación estructurada durante 5 a 10 minutos. Posteriormente, de forma grupal se revisaba la prueba y se discutían los resultados y su interpretación. El ejercicio usado en las evaluaciones inicial y final fue el mismo, bajo el mismo proceso de interpretación del taller. Para fines de calificación, a la interpretación correcta del patrón ventilatorio se le daba un valor de 5 puntos y otros 5 puntos para la graduación correcta del grado de gravedad, para un puntaje total de 20 puntos, sumando los 10 puntos del proceso de graduación de calidad.

\section{Análisis estadístico}

Los datos de los cursos y sus participantes, incluyendo la información de las encuestas y de las evaluaciones, fueron integrados en una hoja cálculo y analizados en el programa de análisis estadístico Stata versión $12 .{ }^{17}$ Para la presentación de los datos se usaron proporciones, promedios y desviaciones estándar. Para la comparación de los puntajes totales de las evaluaciones inicial y final, las cuales fluctuaron entre 0 y 100 , se usó la prueba $t$ pareada. Para la comparación de aciertos iniciales y finales de cada ítem del instrumento de evaluación se usó la prueba de McNemar. Un valor de $\mathrm{p}<$ 0.05 se consideró de significancia estadística y en todos los casos se realizó la corrección por comparaciones múltiples de Bonferroni. ${ }^{18} \mathrm{Al}$ final, se formularon modelos de regresión múltiple para identificar qué características basales predecían el puntaje final, tanto de los conocimientos teóricos como de los prácticos evaluados en los participantes.

\section{Consideraciones éticas}

Todos los participantes acudieron por invitación y los investigadores no tuvieron acceso a datos personales de los mismos. Las encuestas y evaluaciones del curso también fueron por invitación por escrito, así como participación voluntaria y anónima.

\section{RESULTADOS}

Entre marzo de 2007 y agosto de 2009 se organizaron un total de 120 cursos en 30 estados de la República Mexicana y la Ciudad de México, con excepción de Campeche y Quintana Roo. Todos los cursos fueron impartidos en día sábado de 9:00 a 14:00 horas. El total de participantes registrados fue de 2,486 médicos, sin embargo, sólo 1,343 (54\%) completaron el curso y sus evaluaciones correspondientes. En la tabla 1 se resumen los datos obtenidos de la encuesta de práctica profesional aplicada al inicio del curso. La edad promedio de los participantes fue de 44.6 años, con una desviación estándar de 11.0; 592 participantes de los cuales $44.1 \%$ fueron mujeres y $71.3 \%$ fueron médicos generales y 
médicos familiares, el resto fueron médicos especialistas no neumólogos. El 81\% tenían cinco o más años de práctica profesional y el $44.8 \%$ tenían 20 o más años, mientras que

Tabla 1: Características basales de los 1,343 participantes de los cursos de espirometría.

\begin{tabular}{|c|c|c|c|}
\hline & \multicolumn{3}{|c|}{$\%$} \\
\hline Hombre/mujer & \multicolumn{3}{|c|}{$55.9 / 44.1$} \\
\hline $\begin{array}{l}\text { Edad, años } \\
30 \text { o más } \\
40 \text { o más } \\
50 \text { o más }\end{array}$ & & $\begin{array}{l}88.1 \\
67.1 \\
38.0\end{array}$ & \\
\hline $\begin{array}{l}\text { Formación profesional } \\
\text { Medicina general y } \\
\text { familiar } \\
\text { Medicina interna } \\
\text { Pediatría } \\
\text { Otras especialidades }\end{array}$ & & $\begin{array}{r}71.3 \\
6.6 \\
4.4 \\
17.7\end{array}$ & \\
\hline $\begin{array}{l}\text { Años en consultorio } \\
5 \text { o más } \\
10 \text { o más } \\
20 \text { o más }\end{array}$ & \multicolumn{3}{|c|}{$\begin{array}{l}81.0 \\
69.2 \\
44.8\end{array}$} \\
\hline $\begin{array}{l}\text { Pacientes atendidos por } \\
\text { semana } \\
10 \text { o más } \\
26 \text { o más } \\
51 \text { o más }\end{array}$ & \multicolumn{3}{|c|}{$\begin{array}{l}91.7 \\
59.9 \\
25.9\end{array}$} \\
\hline $\begin{array}{l}\text { Pacientes atendidos por } \\
\text { asma o EPOC en un mes } \\
\text { Al menos uno } \\
5 \text { o más } \\
10 \text { o más }\end{array}$ & \multicolumn{3}{|c|}{$\begin{array}{l}95.4 \\
53.8 \\
43.3\end{array}$} \\
\hline $\begin{array}{l}\text { Solicitudes mensuales } \\
\qquad \begin{array}{l}\text { Al menos } 1 \\
5 \text { o más } \\
10 \text { o más }\end{array}\end{array}$ & $\begin{array}{l}\text { Espirome- } \\
\text { trías } \\
55.9 \\
13.3 \\
7.8\end{array}$ & $\begin{array}{c}\text { Electrocar- } \\
\text { diogramas } \\
87.7 \\
29.9 \\
16.5\end{array}$ & $\begin{array}{c}\text { Gluce- } \\
\text { mias } \\
98.5 \\
78.2 \\
57.8\end{array}$ \\
\hline $\begin{array}{l}\text { Define correctamente el } \\
\text { diagnóstico de diabetes } \\
\text { con base en glucemia }\end{array}$ & \multicolumn{3}{|c|}{42.7} \\
\hline $\begin{array}{l}\text { Identifica correctamente } \\
\text { los componentes del } \\
\text { electrocardiograma }\end{array}$ & \multicolumn{3}{|c|}{72.4} \\
\hline \multicolumn{4}{|c|}{$\begin{array}{l}\text { Método de diagnóstico de preferencia para la enfermedad } \\
\text { pulmonar obstructiva crónica (EPOC) }\end{array}$} \\
\hline $\begin{array}{l}\text { Espirometría simple } \\
\text { Espirometría con bronco } \\
\text { Radiografía de tórax } \\
\text { Gasometría arterial } \\
\text { Realiza diagnóstico clínic } \\
\text { No contestó }\end{array}$ & lilatador & $\begin{array}{r}28.4 \\
37.2 \\
9.3 \\
0.9 \\
8.3 \\
16.1\end{array}$ & \\
\hline
\end{tabular}

91.7\% refirió atender 10 o más pacientes (respiratorios y no respiratorios) por semana y $59.9 \%$ más de 25 pacientes. En cuanto al uso regular de espirometría, sólo $55.9 \%$ de los médicos solicitaban al menos una espirometría mensual, comparados con $87.7 \%$ y $98.5 \%$ quienes solicitaban, electrocardiogramas y glucemia, respectivamente; $65.6 \%$ de los médicos reconocieron a la espirometría como el método de primera elección para el diagnóstico de EPOC, pero sólo el $37 \%$ lo definieron de forma correcta como espirometría con broncodilatador. Además, más del 10\% de los médicos consideraron otros métodos diagnósticos de primera elección, como la radiografía de tórax (9.3\%) y la gasometría arterial (1\%) mientras que $8.3 \%$ consideraron que el diagnóstico era clínico.

En una escala de 0 a 100, se observó un cambio significativo en los valores promedios, tanto en conocimientos teóricos (35.8 vs. $62.5 \%, \mathrm{p}<0.01)$ como prácticos $(8.8$ vs. $67 \%, \mathrm{p}<0.01)$. Todos los ítems mostraron mejoría significativa en los valores obtenidos (tabla 2). El cambio observado en la competencia para diagnosticar obstrucción al flujo aéreo grave mostró un cambio de 11.1 a 81.2\% de interpretación correcta. Estos cambios no se deben a las características iniciales de los participantes con los cambios observados, dado que se trata de un análisis antes-después.

En la tabla 3 se muestran los predictores del puntaje final obtenido. En el modelo de regresión múltiple formulado para los conocimientos teóricos, obtuvieron mayores puntajes los médicos con alguna especialidad, aquellos con mayor número de pacientes atendidos a la semana, quienes usaban de forma más regular la espirometría (por lo menos una vez al mes) y aquellos con mejores conocimientos para diagnóstico de diabetes, mientras que los médicos con edad de 40 años o más tuvieron menores puntajes. En cambio, en el modelo regresión múltiple formulado para los conocimientos prácticos, se observaron menores puntajes para los médicos con edad mayor o igual a 50 años, con 10 o más años de consultorio y quienes atendían a cinco o más pacientes con asma o EPOC. Por otro lado, los médicos que solicitaban al menos una espirometría al mes y aquellos con mejores conocimientos para diagnóstico de diabetes tuvieron mayores puntajes en la evaluación de conocimientos prácticos.

\section{DISCUSIÓN}

Este es el primer estudio en América Latina (del que tenemos conocimiento) que describe la planeación, estructuración y el impacto de un curso-taller presencial, enfocado en mejorar la competencia médica en la interpretación de la espirometría. Los principales resultados de este estudio son: 1) un bajo nivel de conocimientos teóricos y prácticos con respecto a las indicaciones, definiciones e interpretación de la espirometría en profesionales médicos, en su mayoría de 
Tabla 2: Cambio en los ítems de la evaluación del conocimiento en los 1,343 participantes de los cursos de espirometría.

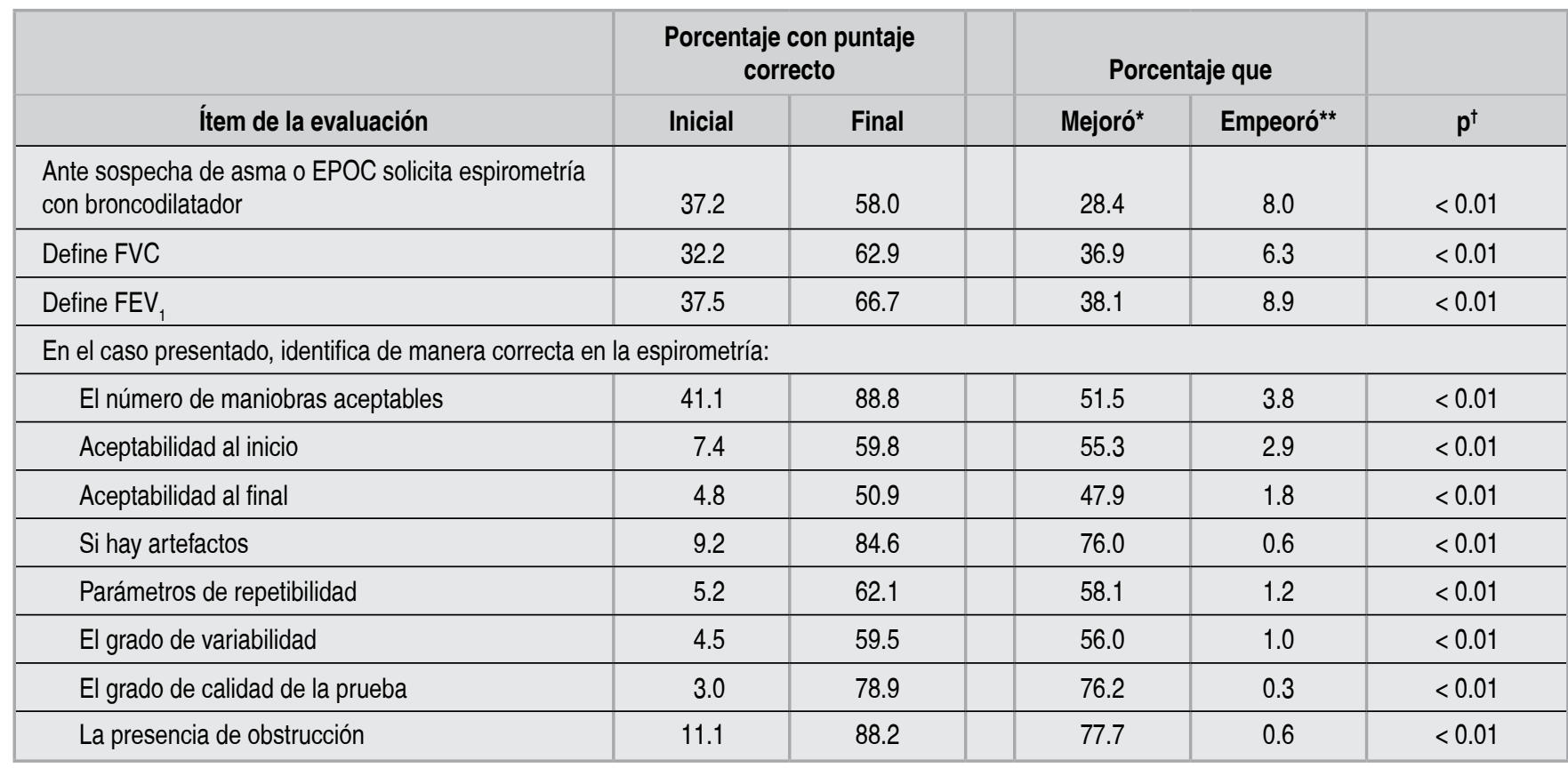

*Mejoró = Ítem contestado de modo incorrecto al inicio del curso y correcto al final del mismo. *^Empeoró = Ítem contestado de modo correcto al inicio del curso e incorrecto al final del mismo.

† Prueba de McNemar con ajuste para comparaciones múltiples de Bonferroni, para las 11 comparaciones presentadas en la tabla. EPOC: enfermedad pulmonar obstructiva crónica, FVC: capacidad vital forzada, $\mathrm{FEV}_{1}$ : volumen espiratorio forzado en un segundo.

atención primaria; 2) una mejoría sustancial y significativa en los conocimientos y en la competencia médica para interpretación de la espirometría, posterior al curso-taller y 3) la identificación de variables como el contar con una especialidad médica, una menor edad, mayor uso de la prueba e incremento de la actividad profesional, medida como número de pacientes atendidos, asociadas a un mejor puntaje obtenido en las evaluaciones de los asistentes.

En este estudio, aun cuando se trataba de un grupo selecto de médicos de práctica profesional privada, que atendían de manera regular pacientes con asma y EPOC, encontramos que sólo dos terceras partes reconocieron, antes de tomar el curso, a la espirometría como método diagnóstico de elección de la EPOC y sólo 37\% definieron de forma acertada a la espirometría con broncodilatador como la prueba de elección. Sólo $11.1 \%$ de los médicos fueron capaces de diagnosticar obstrucción al flujo aéreo grave en un ejercicio de interpretación espirométrica. Es bien conocido que el acceso a la espirometría, así como los conocimientos necesarios para su práctica, uso e interpretación por parte de profesionales médicos, no especializados en el área respiratoria, es insuficiente. Si bien, el uso clínico de la espirometría fue descrito en 1846 por John Hutchinson ${ }^{19}$ tradicionalmente ha sido utilizada sólo por especialistas respiratorios. No fue sino hasta la década de 1990, cuando el estudio longitudinal de salud pulmonar de los Estados Unidos demostró la caída progresiva del flujo aéreo, como la base fisiopatológica más característica de la EPOC..$^{20}$ Asimismo, la Encuesta Nacional de Nutrición y Salud de Estados Unidos (1988-1994) reveló una proporción muy elevada de neumopatía obstructiva no diagnosticada. ${ }^{21}$ De forma similar, el asma es la enfermedad pulmonar más frecuente y su diagnóstico exacto requiere de la demostración de obstrucción variable al flujo aéreo..$^{7-9}$ Este contexto, aunado al incremento progresivo en la morbimortalidad de estas enfermedades, ha obligado al uso más generalizado de la prueba, iniciando los programas de implementación de espirometría en el primer nivel de atención. ${ }^{12,22}$

En contraste con el entrenamiento médico para el uso y la ejecución de pruebas diagnósticas como la medición de la presión arterial, el electrocardiograma y la glucometría, el entrenamiento médico en espirometría es todavía muy insuficiente y no estandarizado, tanto en las escuelas de medicina como en los programas de educación médica continua. Asimismo, históricamente ha existido un mito de que se trata de una prueba costosa, compleja y difícil de interpretar. Los avances técnicos en los dispositivos y en los estándares internacionales para su realización han permitido que ésta sea mucho más accesible y confiable para el diagnóstico, así como simple en sus estrategias de interpretación. Este estudio demostró que un curso presencial de sólo cinco horas, estructurado de forma estandarizada y enfocado en primera instancia a fortalecer la competencia médica en la interpretación de la prueba, permitió que la 
gran mayoría de los médicos fueran capaces de identificar el diagnóstico obstrucción al flujo aéreo. También abarcó entrenamiento en la interpretación de todos los patrones ventilatorios (normal, obstructivo y restrictivo), los grados de gravedad de la obstrucción, la respuesta al broncodilatador y la integración con el diagnóstico clínico, principalmente de asma y EPOC. Sin embargo, para fines prácticos y de tiempo sólo se consideró la evaluación interpretativa de la obstrucción al flujo.

Se puede considerar que una limitación de este estudio es que su implementación se realizó hace algunos años; sin embargo, no esperamos que haya un cambio significativo en el conocimiento médico a nivel primario de atención. Sólo recién (en el año 2014) la Secretaría de Salud inició un programa oficial para la prevención y control de las enfermedades respiratorias, ${ }^{23}$ mismo que ha incluido un proyecto de capacitación e implementación de la espirometría en el primer nivel de atención y con cobertura nacional. Una limitación adicional es que el CIE fue posible gracias a un patrocinador externo y enfocado a médicos de práctica profesional privada con interés en enfermedades respiratorias y en el uso clínico de la espirometría. Los resultados, no necesariamente reflejan otros ambientes de atención médica primaria, como los de instituciones públicas. El CIE es una propuesta para

Tabla 3: Predictores del puntaje final obtenido por los 1,343 participantes de los cursos de espirometría.

\begin{tabular}{|c|c|}
\hline Característica basal & Coeficiente $^{*}$ \\
\hline \multicolumn{2}{|l|}{ Conocimientos teóricos } \\
\hline Edad de 40 años o más & -12.6 \\
\hline Tiene especialidad & 10.3 \\
\hline Atiende a la semana a 5 o más pacientes & 4.1 \\
\hline $\begin{array}{l}\text { Define correctamente el diagnóstico de } \\
\text { diabetes con base en glucemia }\end{array}$ & 7.6 \\
\hline Solicita al menos una espirometría al mes & 5.9 \\
\hline Intercepto & 60.5 \\
\hline \multicolumn{2}{|l|}{ Conocimientos prácticos } \\
\hline Edad de 50 años o más & -7.6 \\
\hline Con 10 o más años de consultorio & -7.0 \\
\hline $\begin{array}{l}\text { Atiende al mes a } 5 \text { o más pacientes con } \\
\text { asma o EPOC }\end{array}$ & -2.8 \\
\hline $\begin{array}{l}\text { Define correctamente el diagnóstico de } \\
\text { diabetes con base en glucemia }\end{array}$ & 2.9 \\
\hline Solicita al menos una espirometría al mes & 4.1 \\
\hline Intercepto & 72.7 \\
\hline
\end{tabular}

${ }^{\star}$ Todos los coeficientes son estadísticamente significativos a un nivel alfa de 0.05 . EPOC: enfermedad pulmonar obstructiva crónica. el entrenamiento médico; pero aún resulta muy limitado para las necesidades de educación continua en los servicios de atención médica de primero y segundo nivel de nuestro país. Sin embargo, la disponibilidad y el formato de las herramientas educativas, el manual, las presentaciones y el video, son materiales ya disponibles que pueden ser implementados de forma digital como un curso en línea que podría ser completado y aprobado en pocas horas por cualquier médico o estudiante de medicina para que adquiera esta competencia básica para el ejercicio profesional moderno. No obstante, es de tomar en cuenta que la metodología de aprendizaje implementada en el taller fue diseñada con base en el aprendizaje colaborativo y social, en el que al reunir personal de salud con diversas experiencias y niveles de conocimiento permitieron el aprendizaje en el tránsito de la zona de desarrollo real a la zona de desarrollo próximo y al posible..$^{24,25}$

\section{CONCLUSIONES}

Este estudio demostró que los conocimientos y la competencia médica sobre las indicaciones, el uso e interpretación de la espirometría en médicos de atención primaria en México es bastante insuficiente con respecto a lo que representa la necesidad en el diagnóstico y manejo de los enfermos pulmonares crónicos, en especial aquellos con asma y EPOC. Un programa educativo teórico-práctico de pocas horas puede ser en extremo eficiente para mejorar la competencia médica en el uso e interpretación de la prueba. La implementación en línea de este programa podría ser una opción para facilitar el acceso al entrenamiento médico estandarizado en la carrera de medicina y en los programas de educación médica continua. Siempre y cuando la plataforma educativa favorezca la interacción con los alumnos, de tal suerte que puedan desarrollar su aprendizaje.

\section{REFERENCIAS}

1. Instituto Nacional de Estadística Geografía e Informática. Registros de mortalidad. [Consultado: 27 de julio de 2017]. Disponible en: http://www.inegi.org.mx/est/contenidos/proyectos/registros/vitales/ mortalidad/tabulados/ConsultaMortalidad.asp.

2. Menezes AM, Perez-Padilla R, Jardim JR, et al.; PLATINO Team. Chronic obstructive pulmonary disease in five Latin American cities (the PLATINO study): A prevalence study. Lancet 2005;366(9500):1875-1881.

3. Regalado J, Pérez Padilla JR. Epidemiología del asma. En: Salas Hernández J, Chapela Mendoza R, Vargas MH, editores. Asma y condiciones especiales. México: PyDESA; 2015.p.1-25.

4. Lamprecht B, Soriano JB, Studnicka M, et al.; BOLD Collaborative Research Group, the EPI-SCAN Team, the PLATINO Team, and the PREPOCOL Study Group. Determinants of underdiagnosis of COPD in national and international surveys. Chest 2015;148(4):971-985. doi: 10.1378/chest.14-2535. 
5. Pérez-Padilla R. Would widespread availability of spirometry solve the problem of underdiagnosis of COPD? Int J Tuberc Lung Dis 2016;20(1):4. doi: 10.5588/ijtld.15.0893.

6. Perez-Padilla R, Torre Bouscoulet L, Vázquez García JC. Implementing a spirometry program. Int J Tuberc Lung Dis 2016;20(9):1142. doi: 10.5588/ijtld.16.0512.

7. Guía Mexicana de Asma. Neumol Cir Torax 2017;76(suppl 1):s1-s136.

8. Global Strategy for Asthma Management and Prevention, Global Initiative for Asthma (GINA) 2017 Update. [Consultado: 28 de junio de 2017]. Disponible en: http://ginasthma.org/.

9. Global Initiative for Chronic Obstructive Lung Disease (GOLD) Global strategy for the diagnosis, management and prevention of COPD, GOLD 2017. [Consultado: 28 de junio de 2017]. Disponible en: http:// goldcopd.org/gold-2017-global-strategy-diagnosis-managementprevention-copd/.

10. Miller MR, Hankinson J, Brusasco V, et al. Standardization of spirometry. Eur Respir J 2005;26(2):319-338.

11. Pellegrino R, Viegi G, Brusasco V, et al. Interpretative strategies for lung function tests. Eur Respir J 2005;26(5):948-968.

12. Ferguson GT, Enright PL, Buist AS, Higgins MW. Office spirometry for lung health assessment in adults: a consensus statement from the National Lung Health Education Program. Respir Care 2000;45(5):513-530.

13. Levy ML, Quanjer PH, Booker R, Cooper BG, Holmes S, Small IR.; General Practice Airways Group. Diagnostic spirometry in primary care: Proposed standards for general practice compliant with American Thoracic Society and European Respiratory Society recommendations. Prim Care Respir J 2009; 18(3):130-147. doi: 10.4104/pcrj.2009.00054.

14. Coates A L, Graham BL, McFadden RG, et al. Spirometry in primary care. Can Respir J 2013;20(1):13-21.

15. Pérez PJR, Vázquez GJC. Manual de entrenamiento en espirometría. México: Asociación Latinoamericana del Tórax (ALAT); 2006.

16. Vázquez GJC, Pérez PJR. Manual para el uso y la interpretación de la espirometría por el Médico. México: Asociación Latinoamericana del Tórax (ALAT); 2007.

17. StataCorp. 2011. Stata: Release 12. Statistical Software. College Station, TX: StataCorp LP.
18. Pagano M, Gauvreau K. Principles of biostatistics. CA: Duxbury; 2000.

19. Hutchinson J. On the capacity of the lungs, and on the respiratory functions, with a view of stablishing a precise and easy method of detecting disease by the spirometer. Med Chir Trans 1846;29:137-252.

20. Anthonisen NR, Connett, JE, Kiley JP, et al. Effects of smoking intervention and the use of an inhaled anticholinergic bronchodilator on the rate of decline of FEV 1 . The Lung Health Study. JAMA 1994;272(19):1497-1505.

21. Mannino DM, Gagnon RC, Petty TL, Lydick E. Obstructive lung disease and low lung function in adults in the United States: data from the National Health and Nutrition Examination Survey, 1988-1994. Arch Intern Med 2000;160:1683-1689.

22. Petty TL, Weinmann GG. Building a national strategy for the prevention and management of and research in chronic obstructive pulmonary disease. National Heart, Lung, and Blood Institute Workshop Summary. JAMA 1997;277(3):246-253.

23. Secretaría de Salud. Prevención y control de las enfermedades respiratorias e influenza. Programa Sectorial de Salud 2013-2018. México: SSA; 2014.

24. Ledesma AM. Análisis de la Teoría de Vigotsky para la reconstrucción de la inteligencia social. Cuenca, Ecuador: Universidad Católica de Cuenca; 2014. p.104.

25. Arancibia V, Herrera P, Strasser K. Psicología de la educación. $2^{\text {a }}$ ed. México, D.F.: Alfaomega, Universidad Católica de Chile; 1999. p. 280.

Financiamiento: Este estudio fue financiado en su totalidad y sin restricciones por Boehringer Ingelheim México. Dicho financiamiento abarcó el diseño y la edición de los materiales educativos, así como la organización de los cursos. El patrocinador no tuvo ninguna influencia en el contenido de los materiales ni en la impartición de los cursos, los cuales no incluyeron información comercial.

Conflicto de intereses: Los autores declaran no tener conflicto de intereses. 